Marjana Šifrar Kalan

Universidad de Ljubljana

\title{
DISPONIBILIDAD LÉXICA EN ESPAÑOL COMO LENGUA EXTRANJERA: EL COTEJO DE LAS INVESTIGACIONES EN ESLOVENIA, SALAMANCA Y FINLANDIA
}

Palabras clave: competencia léxica, disponibilidad léxica, léxico fundamental, español como lengua extranjera

\section{Introducción}

La competencia léxica es parte fundamental del conocimiento lingüístico y una herramienta cognitiva que nos permite comunicar en una lengua. El léxico, además de ser una manifestación antropológica, es la parte más inestable e íntima de la lengua que puede variar mucho dependiendo de factores muy variados. Si se trata de una lengua extranjera es necesario saber cuál es el léxico fundamental que nos permite desenvolvernos en las situaciones comunicativas más frecuentes. Algunas investigaciones sobre el vocabulario ya pusieron de manifiesto que, habitualmente, el hombre corriente no emplea más de dos mil palabras; el hombre culto, por su parte, se sirve de unos cuatro o cinco mil vocablos (ver Carcedo González, 2000: 11; Nation, 1990: 11, 2001: 9). Benítez Pérez (1994a: 10) señala que «según los estudiosos de frecuencias léxicas, las primeras 1000 palabras más usadas en español abarcan cerca del $85 \%$ del contenido del léxico de cualquier texto no especializado». Cabría añadir lo que ya han demostrado otras investigaciones realizadas sobre lenguas tan lejanas como el chino, el japonés, el francés, y es el hecho de que exista una constante entre muchas lenguas del mundo cuyo vocabulario fundamental varía de 1500 a 2000 palabras sin pasar nunca de esta cifra. De aquí la importancia de escoger cuidadosamente el vocabulario fundamental que debemos enseñar. Según López Morales (1999: 20) el léxico fundamental de una lengua está formado por el léxico básico y el léxico disponible. El léxico básico abarca las palabras más usuales o frecuentes de una comunidad, que se caracterizan por un alto grado de estabilidad o permanencia. Esto les permite que aparezcan con mucha frecuencia en todo tipo de discursos con independencia del tema tratado. En cambio, el léxico disponible está compuesto por aquellas palabras que sólo aparecen en situaciones precisas, es decir, cuando el tema de conversación las requiere. De este modo queda claro que las palabras disponibles son palabras muy conocidas, pero no muy frecuentes, a causa de que es necesario tocar un tema específico para que aparezcan (López Chávez, 1994: 69). López Morales (1999: 11) a su vez aduce que se trata de un «caudal léxico utilizable en una situación comunicativa dada», donde por léxico utilizable se entiende el léxico disponible. Los trabajos de disponibilidad léxica son entonces aquellas investigaciones que tienen como objetivo principal conocer el léxico disponible de un grupo determinado de hablantes. «De esta manera el rango de disponibilidad léxica va en función de las palabras que acuden antes a la mente del hablante en relación con un tema determinado» (Galloso Camacho, Prado Aragonés, 2005: 370). 
El objetivo del presente trabajo es comparar cuantitativa y cualitativamente la disponibilidad léxica de un grupo de alumnos eslovenos de español como lengua extranjera con otras dos investigaciones sobre la disponibilidad léxica en ELE, una llevada a cabo en Finlandia (Carcedo González, 2000) y otra en Salamanca (Samper Hernández, 2002), y consecuentemente detectar las diferencias o similitudes léxicas entre los hablantes extranjeros de español. Seguimos el mismo objetivo que ya expuso Samper Hernández (2002: 17) e igualmente consideramos que «esta comparación podría resultar de gran provecho para el reconocimiento del aprendizaje/adquisición del español como lengua extranjera y puede abrir una vía de investigación que podremos desarrollar en un futuro».

\section{Aplicación de la disponibilidad léxica}

Aunque el objetivo de los primeros proyectos sobre disponibilidad léxica fue determinar el léxico fundamental del francés y lograr su rápida difusión como lengua extranjera en las ex-colonias, muy pronto se comenzaron a aprovechar los datos que la disponibilidad léxica ofrecía para la enseñanza de una de las lenguas de una comunidad bilingüe (ver Carcedo González, 2000) y a partir del año 1973 (López Morales) para el español como lengua materna hasta llegar a la versión actual de Humberto López Morales (1999). Las aplicaciones de los métodos de la léxico-estadística han dado lugar a valiosos estudios en el ámbito hispanohablante [por citar algunos de ellos, Alba (1995); Benítez Pérez (1992); Carcedo González (2001); Galloso Camacho (1998); González Martínez (2002); López Chávez (1993); Mateo García (1998); Prado Aragonés y Galloso Camacho (2005); Samper y Hernández (1997); Valencia y Echeverría (1999)].

Afirman Rodríguez Muñoz y Muñoz Hernández (2008) que «a pesar de la evolución que han experimentado las investigaciones sobre disponibilidad léxica en el ámbito hispánico, pocas veces se han sabido aprovechar las nuevas metodologías de análisis léxico y su aplicabilidad a la enseñanza de lenguas extranjeras ». Entre los pocos investigadores que se han dedicado a este campo, podemos distinguir entre aquellos que han aplicado los estudios de disponibilidad léxica de hablantes nativos a la enseñanza de ELE (Benítez Pérez, 1994a; García Marcos y Mateo, 2000), y aquellos lingüistas que han aplicado la metodología de estos trabajos a informantes extranjeros -estudiantes de ELE. En el estudio presente hemos seguido esta segunda vertiente, donde destacan las investigaciones de Alberto Carcedo, quien analiza la disponibilidad léxica de los alumnos de español en Finlandia y la compara con la disponibilidad léxica de los hablantes nativos. Además de sus exhaustivos estudios, aprovecharemos los estudios de Samper Hernández (2002), quien analizó la disponibilidad léxica de 45 estudiantes extranjeros durante su curso de ELE en Salamanca. ${ }^{1}$ Con nuestro acercamiento a la disponibilidad léxica en alumnos eslovenos que aprenden español como lengua extranjera, queremos seguir esta línea de investigaciones aplicada a la enseñanza y el aprendizaje del léxico español. Samper Hernández explica que estas investigaciones nos permiten:

La disponibilidad léxica con informantes de ELE ha sido estudiada también por Sánchez Gómez (2003), Galloso Camacho y Prado Aragonés (2005) y Frey Pereyra (2007) pero no disponemos de todos los resultados para poder cotejarlos en este artículo. 
[...] examinar las distintas fases de aprendizaje del léxico español, descubrir tanto los errores más usuales como las tendencias de los hablantes no nativos a lo largo de este aprendizaje, planificar la enseñanza del léxico o realizar provechosas comparaciones con los listados de las distintas zonas hispanoblantes. (Samper Hernández, 2002: 14)

\section{Método y criterios de muestreo}

Para el estudio de la disponibilidad léxica en el español de alumnos eslovenos hemos seguido muy de cerca las pautas metodológicas comunes del proyecto panhispánico del léxico disponible ${ }^{2}$, aunque hemos planteado algunos cambios que se explican a continuación. En la primera fase de nuestra investigación recogimos la muestra en diferentes Institutos de Educación Secundaria (IES) de Eslovenia donde se enseña español como segunda o tercera lengua extranjera. La muestra de 100 alumnos (de 17 ó 18 años) del final del tercer curso ${ }^{3}$ fue escogida entre casi $4000^{4}$ alumnos que en los 2 años $(2007,2008)$ de recogida de datos estudiaron español.

Las variables que se han tenido en cuenta difieren bastante de las del proyecto panhispánico, ya que analizar las características de una lengua extranjera es diferente del análisis de la lengua materna, además en nuestro caso se trata de una muestra muy homogénea - de la misma edad y de la misma lengua materna. También hemos descartado las variables como el nivel sociocultural, la procedencia rural o urbana, e incluso el sexo, ya que la mayoría de los alumnos que aprenden español en los institutos son chicas. Además el estudio de Samper Hernández (2002) indica, que no existe una relación asociativa.

Los centros de interés contemplados en nuestra muestra son: 1) Partes del cuerpo (CUE), 2) La ropa (ROP), 3) La casa (CAS), 4) Alimentos y bebidas (ALI), 5) La ciudad (CIU), 6) El campo (CAM), 7) Medios de transporte (TRA), 8) Los animales (ANI), 9) Juegos y distracciones (JUE), 10) Profesiones y oficios (PRO), 11) Acciones que normalmente se realizan todos los días. Estamos de acuerdo con López Morales cuando puntualiza que:

[...] a pesar de que los centros sean unos u otros, se mantiene siempre la misma preocupación que ya estaba presente en los autores franceses: ¿cuál sería la mejor forma de encasillar en centros de interés todos los intereses humanos?; ¿con cuántos centros nos obligaría a trabajar una investigación que pretendiera ser exhaustiva? Nadie, que sepamos, ha intentado adelantar respuestas a estos interrogantes. (López Morales, 1999: 33)

Samper Hernández y Carcedo González utilizan los mismos 16 centros que aparecen también en la primera encuesta de Gougenheim en Francia. Sin embargo, por razones prácticas, hemos decidido unir «Las partes de la casa sin muebles» con «Los muebles

2 El proyecto, coordinado e impulsado por H. López Morales, que comenzó en Puerto Rico los trabajos de disponibilidad para el español (López Morales, 1999), ya cuenta con varios léxicos disponibles nacionales y regionales.

3 La muestra se escogió entre los alumnos de ELE con aproximadamente 300 horas de español, estudiándolo sólo en el ámbito escolar. Los informantes tenían un nivel A2 o B1 según el MCER.

4 El número de casi 4000 alumnos se refiere a todos los alumnos que en los IES estudiaron español como segunda o tercera lengua extranjera desde el primer al cuarto año de la secundaria. 
de la casa» en un solo centro de interés: «La casa». Asimismo, hemos eliminado «Los objetos colocados en la mesa para la comida», «La cocina y sus utensilios», «La escuela: muebles y materiales», «Iluminación y calefacción», «Trabajos del campo y del jardín», ${ }^{5}$ es decir, aquellos centros que se han mostrado como menos productivos tanto entre los finlandeses (Carcedo González, 2000: 93) y los estudiantes extranjeros en Salamanca (Samper Hernández, 2002: 48), como también entre los hispanohablantes (López Morales, 1999: 41; Carcedo González, 2001; 58; González Martínez, 2002: 22; Prado Aragonés y Galloso Camacho, 2005: 56). Hemos añadido un nuevo centro de interés: «Acciones que normalmente se realizan todos los días» ${ }^{6}$ para poder realizar en el futuro un estudio sobre los verbos, las colocaciones y las coincidencias léxicas con ciertos manuales de ELE.

Según el procedimiento tipificado para este tipo de estudios, el material lingüístico se ha recogido mediante una prueba de asociación controlada, con once áreas temáticas que actúan como estímulo y listas abiertas, con lo que los resultados dan cabida a cuantas lexías son capaces de producir los sujetos participantes en la prueba en un tiempo límite de dos minutos por centro de interés (campo semántico). Samper Hernández (2002: 21) plantea que a pesar de que los estudiantes no nativos quizá necesiten más tiempo para acordarse de las distintas unidades que conocen, al mismo tiempo disponen de menor cantidad de vocablos, por lo que dos minutos parecen suficientes. De la misma forma, antes de empezar con las asociaciones, fue necesario pedir a los alumnos que escribieran las palabras en el orden que se les ocurrían. De este modo se puede tener en cuenta la posición de la palabra dentro de todas las respuestas, que es el aspecto fundamental de clasificación según la disponibilidad. Igualmente, se les instruyó que escribieran cualquier palabra que se les ocurriera en conexión con el tema, esto es, el título del centro de interés.

Para unificar, eliminar y corregir el léxico recogido hemos seguido los criterios de Samper Hernández (2002) y Carcedo González (2000), y para informatizarlo nos hemos servido del programa DISPOLEX (Universidad de Salamanca) de Bartol y Hernández.

\section{Comparación del vocabulario disponible de estudiantes de español como lengua extranjera}

La comparación parte de tres investigaciones que se llevaron a cabo en Salamanca, España, en Turku, Finlandia y en Ljubljana, Eslovenia. En Salamanca la recogida fue realizada a 45 estudiantes extranjeros de diferentes países; en Finlandia se recopiló el material lingüístico a 350 finlandeses, pero se comparan aquí los resultados de 150 informantes preuniversitarios, ya que los resultados eslovenos parten también de 100 alumnos

\footnotetext{
En el estudio de Salamanca el promedio de unidades por informante en este centro de interés es sólo 3,95, en Finlandia una sola unidad. Se debe esto a varios factores bastante evidentes: este campo semántico no resulta en los manuales de ELE, tampoco es del interés de la mayoría de los jóvenes que aprenden español como lengua extranjera. Estos campos, como señala Izquierdo Gil (2005: 90), son «alejados del universo adolescente o hacen referencia a conceptos cuyo conocimiento no nos parece indispensable para un nivel elemental».

6 Izquierdo Gil (2005: 90) también critica que los 16 centros de interés han desatendido algunos centros de interés tales como las acciones de la vida cotidiana, el carácter, el vocabulario relativo a las relaciones familiares, y algunos más.
} 
preuniversitarios. El estudio comparativo se refiere a los diez centros de interés comunes a las tres investigaciones (hemos dejado atrás «Acciones que normalmente se realizan todos los días», por lo tanto, los resultados de los diez campos léxicos difieren de los obtenidos de los 16 campos o centros léxicos).

\begin{tabular}{|l|c|c|c|}
\hline & $\begin{array}{c}\text { Ljubljana } \\
\text { ESLOVENIA }\end{array}$ & $\begin{array}{c}\text { Salamanca } \\
\text { ESPAÑA }\end{array}$ & $\begin{array}{c}\text { Turku } \\
\text { FINLANDIA }\end{array}$ \\
\hline Número de informantes & 100 & 45 & 150 \\
\hline Nivel de estudio & preuniversitarios & universitarios & preuniversitarios \\
\hline Lengua materna & esloveno & varias & finés/sueco \\
\hline $\begin{array}{l}\text { Promedio de palabras por } \\
\text { informante (10 centros de } \\
\text { interés comunes) }\end{array}$ & $\mathbf{1 2 , 5 3}$ & $\mathbf{1 3 , 6 8}$ & $\mathbf{7 , 2}$ \\
\hline
\end{tabular}

Cuadro 1: Comparación de algunos datos de Eslovenia/Salamanca/Finlandia

El cuadro 1 permite observar primeramente algunos datos generales a los que los siguen las comparaciones cuantitativas. Los promedios de palabra por informante oscilan de un 13,68 en los estudiantes extranjeros de Salamanca hasta un 12,53 en los alumnos eslovenos, y bajan a un 7,2 en los alumnos finlandeses, refiriéndose sólo a los diez centros de interés comunes. Aunque la comparación con la disponibilidad léxica de los hispanohablantes no es el objetivo de este artículo, cabe añadir que la disponibilidad léxica de los informantes no hispanohablantes nativos es menor cuantitativamente, dado que «en los trabajos hispánicos los resultados superan casi siempre el número de 20 unidades por informante». (Samper Hernández, 2002: 46).

\begin{tabular}{|l|c|c|c|c|}
\hline Centro de interés & RANGO & PALABRAS & RANGO & VOCABLOS \\
\hline 01. Partes del cuerpo (CUE) & 7 & 1209 & 11 & 128 \\
\hline 02. La ropa (ROP) & 11 & 986 & 10 & 167 \\
\hline 03. La casa (CAS) & 5 & 1299 & 5 & 290 \\
\hline 04. Alimentos y bebidas (ALI) & 2 & 1544 & 6 & 273 \\
\hline 05. La ciudad (CIU) & 3 & 1434 & 1 & 335 \\
\hline 06. El campo (CAM) & 9 & 1122 & 7 & 272 \\
\hline 07. Medios de transporte (TRA) & 10 & 1078 & 9 & 177 \\
\hline 08. Los animales (ANI) & 8 & 1163 & 8 & 249 \\
\hline 09. Juegos y distracciones (JUE) & 4 & 1401 & 4 & 297 \\
\hline 10. Profesiones y oficios (PRO) & 6 & 1294 & 2 & 321 \\
\hline 11. Acciones de cada día (ACC) & 1 & 1603 & 3 & 307 \\
\hline
\end{tabular}

Cuadro 2: Palabras frente a vocablos en el estudio esloveno 
El cuadro 2 muestra la diferencia entre el número de palabras y el número de vocablos entre los centros de interés en el estudio esloveno. El número de palabras indica el número de palabras totales que han escrito los informantes, mientras que el número de vocablos indica el número de palabras diferentes en cada centro de interés. Como vemos, hay algunas diferencias en el rango entre palabras y vocablos. La mayor diferencia se observa en «Partes del cuerpo», que según las palabras ocupa el séptimo lugar y según los vocablos el último lugar. Esto significa que a pesar de que en este centro de interés hay un número de palabras totales bastante alto, el número de palabras diferentes es bastante menor que en otros centros de interés. Es decir, que varias palabras se repiten entre los informantes, esto es, que los informantes conocen más o menos las mismas palabras en relación con este tema. Del mismo modo también el centro «Alimentos y bebidas» pasa del segundo lugar según las palabras al sexto lugar según los vocablos, lo contrario que con «Profesiones y oficios».

Comparando los rangos de los diez centros comunes eslovenos con los de España (Salamanca) y Finlandia, observamos que la coincidencia es sorprendente: los informantes de ELE de los tres estudios han mostrado mayor productividad de palabras en el campo sobre la comida y bebida, seguido por el campo semántico sobre la ciudad. El centro menos productivo en Eslovenia ha sido la ropa; en Salamanca y en Finlandia coincide el centro de los trabajos del campo y del jardín. En cuanto a los vocablos o palabras diferentes en Eslovenia el campo más variado es la ciudad, mientras que en Salamanca y Finlandia los alimentos y bebidas, mostrándose de nuevo como el campo más productivo, esta vez en vocablos.

\subsection{Los vocablos más disponibles}

\begin{tabular}{|l|l|l|l|l|l|l|}
\hline & C.I. & ESLOVENIA & C.I. & SALAMANCA & C.I. & FINLANDIA \\
\hline 1 & ANI & PERRO $(0.78101)$ & TRA & COCHE $(0.85349)$ & ANI & PERRO $(0.82550)$ \\
\hline 2 & TRA & COCHE $(0.77542)$ & ANI & PERRO $(0.83753)$ & PRO & PROFESOR $(0.80271)$ \\
\hline 3 & ANI & GATO $(0.77275)$ & ANI & GATO $(0.81794)$ & TRA & COCHE $(0.7643)$ \\
\hline 4 & CUE & CABEZA $(0.73379)$ & PRO & PROFESOR $(0.80847)$ & TRA & AUTOBÚS $(0.69947)$ \\
\hline 5 & CUE & MANO $(0.70785)$ & TRA & AUTOBÚS $(0.79015)$ & TRA & TREN $(0.69009)$ \\
\hline 6 & TRA & BICICLETA $(0.68523)$ & TRA & TREN $(0.78347)$ & CUE & MANO $(0.68576)$ \\
\hline 7 & ROP & PANTALÓN $(0.67052)$ & CAM & ÁRBOL $(0.75772)$ & CAS & COCINA $(0.67320)$ \\
\hline 8 & PR0 & PROFESOR $(0.65328)$ & ROP & PANTALÓN $(0.74344)$ & ANI & GATO $(0.67102)$ \\
\hline 9 & TRA & AUTOBÚS $(0.64509)$ & TRA & AVIÓN $(0.72088)$ & CUE & CABEZA $(0.63715)$ \\
\hline 10 & ROP & CAMISA $(0.61255)$ & CIU & CALLE $(0.68909)$ & JUE & FÚTBOL $(0.60923)$ \\
\hline 11 & TRA & AVIÓN $(0.60390)$ & CUE & OJO $(0.68092)$ & TRA & AVIÓN $(0.57374)$ \\
\hline 12 & TRA & TREN $(0.58864)$ & CAS & COCINA $(0.66482)$ & ROP & ZAPATO $(0.56426)$ \\
\hline 13 & CUE & OJO $(0.57408)$ & CUE & CABEZA $(0.64904)$ & ALI & LECHE $(0.54350)$ \\
\hline 14 & JUE & FÚTBOL $(0.52743)$ & ROP & CAMISETA $(0.61751)$ & CUE & OJO $(0.52908)$ \\
\hline
\end{tabular}




\begin{tabular}{|l|l|l|l|l|l|l|}
\hline 15 & ANI & VACA $(0.52285)$ & JUE & FÚTBOL $(0.59405)$ & CIU & CALLE $(0.50586)$ \\
\hline 16 & CAM & VACA $(0.52180)$ & CUE & PIE $(0.57269)$ & TRA & BICICLETA $(0.50570)$ \\
\hline 17 & JUE & BALONCEST0 $(0.51732)$ & CAM & ANIMAL $(0.56042)$ & ANI & CABALL0 $(0.47052)$ \\
\hline 18 & ANI & CABALL0 $(0.49023)$ & ROP & CAMISA $(0.55714)$ & CAS & DORMITORIO $(0.47278)$ \\
\hline 19 & CIU & CALLE $(0.46787)$ & TRA & BICICLETA $(0.54771)$ & ALI & AGUA $(0.46088)$ \\
\hline 20 & COM & AGUA $(0.45926)$ & ANI & VACA $(0.53605)$ & CUE & PIE $(0.44106)$ \\
\hline
\end{tabular}

Cuadro 3: Los 20 vocablos más disponibles en informantes de ELE de Eslovenia, Salamanca y Finlandia (preuniversitarios) de los diez centros comunes. (Los recuadros de color gris señalan las palabras comunes).

El cotejo del cuadro 3 se basa en los vocablos que aparecen en los puestos más altos de cada uno de los diez centros comunes, puesto que el índice de disponibilidad tiene en cuenta tanto el número de apariciones de un determinado vocablo como el lugar en el que se menciona. Sorprende la gran cantidad de coincidencias que se han producido entre las listas de las tres investigaciones, llevadas a cabo en tres partes tan diferentes de Europa. Las palabras con mayor disponibilidad que aparecen en las tres listas suman 12 (marcadas de color gris): perro, coche, gato, cabeza, bicicleta, profesor, autobús, avión, tren, ojo, fútbol, calle. Entre Eslovenia y Salamanca hay 15 palabras en común, al igual que entre Eslovenia y Finlandia, mientras que entre Salamanca y Finlandia hay $14^{7}$. Los casos más sorprendentes son, sin duda, las primeras posiciones donde hay mucha similitud (perro, coche, gato, profesor). También las palabras que no aparecen en alguna de las tres listas aparecerían en las posiciones entre 20 ó 30. Los 20 vocablos más disponibles iguala a los 20 sustantivos más disponibles ya que no se encuentra otra categoría gramatical.

Este cotejo pone de relieve la similar competencia léxica básica que tienen los estudiantes ELE, a pesar de tener lenguas maternas tan diversas y vivir en culturas muy diferentes. Se podría añadir muchas diferencias más, pero también hay que admitir que todos los informantes tienen algo en común, no sólo el hecho de estudiar todos español como lengua extranjera sino también que son adultos jóvenes que viven en la civilización occidental del siglo XXI. Y en el mundo global de hoy esto es un hecho que hay que tomar en cuenta.

Desde el punto de vista de la adquisición del léxico, se nos ocurren las siguientes preguntas al observar los vocablos del cuadro 3: ¿Los informantes aprendieron estos vocablos en su fase inicial de aprendizaje de español? ¿Los manuales de nivel A1 según MCER contienen todos estos vocablos o hay que buscar alguno en el nivel A2 o tal vez B1? ¿Los alumnos o estudiantes de ELE al obtener el nivel A1 conocen todos estos vocablos?

\footnotetext{
Como aquí se cotejan sólo diez centros comunes, los resultados difieren un poco de los presentados por Samper Hernández (2002: 53) donde se comparan los 16 centros del estudio en Salamanca y Finlandia. En aquel listado de los 20 vocablos más disponibles, coinciden 15 palabras.
} 
La afirmación de Carcedo González (2000: 113) de que «el léxico español disponible de los estudiantes finlandeses parece integrar unidades más próximas a una lengua estándar en el que las voces propiamente americanas pocas veces están presentes» podría aplicarse a otros estudiantes extranjeros, ya que ellos eligen, por ejemplo, coche frente a carro, autobús frente a guagua etc.

\subsection{Los vocablos más disponibles según los diez centros de interés}

A continuación la comparación se limita a las diez primeras posiciones de las diez áreas temáticas ya mencionadas. En el centro de interés «Partes del cuerpo» en el estudio esloveno encontramos 1209 palabras y 128 vocablos, en Salamanca 735 palabras y 75 vocablos, y en Finlandia 1186 palabras y 61 vocablos. En el caso de Finlandia vemos que una mayor producción de palabras no implica necesariamente un mayor número de vocablos. Los tres grupos tienen 6 palabras en común (marcados de color gris): cabeza, mano, ojo, dedo, nariz, boca. Entre Eslovenia y Salamanca hay 7 palabras comunes, entre Eslovenia y Finlandia hay 8, y entre Salamanca y Finlandia también 7. Se puede concluir que aquí hay más similitudes cualitativas que cuantitativas. En el listado esloveno, además de los sustantivos que indican partes del cuerpo, encontramos en este centro también varios adjetivos (el 15\% de todos los vocablos de este centro) que los describen como alto, gordo, algunos verbos (el 20\% son verbos o sintagmas verbales) como correr, mover, mirar, palabras en relación con la salud dolor, medicamento, médico y también palabras como mestizo y negro.

\begin{tabular}{|l|l|l|l|}
\hline RANGO & ESLOVENIA $(12.09)^{8}$ & SALAMANCA $(16.33)$ & FINLANDIA (7.9) \\
\hline 1 & CABEZA & OJO & MANO \\
\hline 2 & MANO & CABEZA & CABEZA \\
\hline 3 & OJO & PIE & OJO \\
\hline 4 & PIERNA & PIERNA & PIE \\
\hline 5 & DEDO & MANO & PELO \\
\hline 6 & NARIZ & NARIZ & ESPALDA \\
\hline 7 & PELO & BRAZO & BOCA \\
\hline 8 & RODILLA & DEDO & NARIZ \\
\hline 9 & ESPALDA & OREJA & ESTÓMAGO \\
\hline 10 & BOCA & BOCA & DEDO \\
\hline
\end{tabular}

Cuadro 4: Partes del cuerpo: comparación Eslovenia/Salamanca/Finlandia

También en el campo sobre «La ropa» hay bastante coincidencia entre el número de palabras en Eslovenia (986) y Finlandia (907), pero es Eslovenia la que agrupa un

8 En los cuadros a partir del número 4 se proporcionan también los promedios por informante (ver Samper Hernández, 2002: 45 y Carcedo González, 2000a: 101). 
mayor número de vocablos (167). Los tres grupos tienen 7 palabras en común. Entre Eslovenia y Salamanca hay 8, lo mismo que entre Eslovenia y Finlandia y entre Salamanca y Finlandia. El número tan grande de palabras comunes disponibles se debe a que el campo semántico es bastante cerrado, mucho más que, por ejemplo, centros más abiertos como «La ciudad»o «El campo» que pueden estimular asociaciones muy variadas.

Como ya mencionado anteriormente, un mayor número de informantes extranjeros optan por la variante peninsular que por los anglicismos. Así la palabra vaquero aparece en la posición 8 en Eslovenia, en la posición 13 en Salamanca y 3 en Finlandia, mientras que la variante americana jeans cobra un índice de disponibilidad sensiblemente inferior (posición 46 en Salamanca, 21 en Finlandia, 38 en Eslovenia). La misma preferencia se revela en el caso de camiseta (posición 4 en Eslovenia, 2 en Salamanca, 11 en Finlandia) y $t$-shirt (32 en Eslovenia, 57 en Salamanca).

\begin{tabular}{|l|l|l|l|}
\hline RANGO & ESLOVENIA (9.86) & SALAMANCA (11.66) & FINLANDIA (6.00) \\
\hline 1 & PANTALÓN & PANTALÓN & ZAPATO \\
\hline 2 & CAMISA & CAMISETA & PANTALÓN \\
\hline 3 & ZAPATO & CAMISA & VAQUERO \\
\hline 4 & CAMISETA & ZAPATO & BLUSA \\
\hline 5 & FALDA & CHAQUETA & CAHQUETA \\
\hline 6 & JERSEY & FALDA & JERSEY \\
\hline 7 & VESTIDO & JERSEY & CAMISA \\
\hline 8 & VAQUERO & ABRIGO & VESTIDO \\
\hline 9 & CORBATA & CALCETÍN & FALDA \\
\hline 10 & CHAQUETA & VESTIDO & CALCETÍN \\
\hline
\end{tabular}

Cuadro 5: La ropa: comparación Eslovenia/Salamanca/Finlandia

En el centro «La casa» la palabra más disponible y también más frecuente es cocina. Sin embargo, el grado de disponibilidad de la palabra cocina en Eslovenia es bastante menor (0.37806 en Eslovenia; 0.66482 en Salamanca; 0.67320 en Finlandia). Esto se debe al hecho de que la investigación se llevó a cabo sobre 11 centros de interés, y se omitieron centros más específicos como «Los muebles de la casa», «La cocina y sus utensilios», por lo tanto aparecen en la lista de las palabras más disponibles objetos como cama, mesa, silla, sofá, televisión, ordenador, y la asociación de familia con la casa. El tema de «La casa» en Eslovenia resulta más abierto que en las otras dos investigaciones, donde se llama «Partes de la casa». 


\begin{tabular}{|l|l|l|l|}
\hline RANGO & ESLOVENIA $(12.99)$ & SALAMANCA $(10.11)$ & FINLANDIA $(5.3)$ \\
\hline 1 & COCINA & COCINA & COCINA \\
\hline 2 & CAMA & BAÑO & DORMITORIO \\
\hline 3 & MESA & HABITACIÓN & CUARTO DE BAÑO \\
\hline 4 & FAMILIA & SALÓN & VENTANA \\
\hline 5 & SILLA & PUERTA & PUERTA \\
\hline 6 & SOFÁ & VENTANA & BALCÓN \\
\hline 7 & VENTANA & DORMITORIO & CUARTO DE ESTAR \\
\hline 8 & TELEVISIÓN & PARED & BAÑO \\
\hline 9 & PUERTA & SUELO & GARAJE \\
\hline 10 & ORDENADOR & BALCÓN & ASEO \\
\hline
\end{tabular}

Cuadro 6: La casa: comparación Eslovenia/Salamanca/Finlandia

En el centro más productivo «Alimentos y bebidas» hay menos palabras comunes en la lista de las diez más disponibles que en los centros «Las partes del cuerpo» y «La ropa». Si miramos el cuadro 7, vemos que 4 de las palabras se repiten: agua, cerveza, carne y pan. Es interesante que la comida nacional de España paella ocupe el tercer lugar entre los alumnos de Eslovenia, mientras que entre los estudiantes en Salamanca la encontramos en el lugar 63 y en Finlandia en el lugar 13. Más o menos la mitad son bebidas y la otra mitad comida. Entre los 198 vocablos de Salamanca no aparece ni un verbo o adjetivo. ¿Se habrán eliminado en la fase de unificación de los datos, o los estudiantes de verdad no asociaron la comida y la bebida con los verbos o adjetivos? Tanto en la lista finlandesa como en la salmantina, los adjetivos aparecen sólo como complemento de sustantivo, por ejemplo vino blanco, pollo asado. El primer adjetivo de la lista eslovena que aparece solo, sin sustantivo, es gordo en la posición 63. Entre los 273 vocablos de la lista eslovena aproximadamente el $8 \%$ son verbos o sintagmas verbales. Los verbos más disponibles son: comer en la posición 13, beber en la 26, cocinar en la 30 .

\begin{tabular}{|l|l|l|l|}
\hline RANGO & ESLOVENIA (15.44) & SALAMANCA (20.42) & FINLANDIA (12.4) \\
\hline 1 & AGUA & AGUA & LECHE \\
\hline 2 & CERVEZA & ZUMO & AGUA \\
\hline 3 & PAELLA & CARNE & CERVEZA \\
\hline 4 & VINO & CERVEZA & VINO \\
\hline 5 & ZUMO & PAN & CARNE \\
\hline 6 & FRUTA & LECHE & PAN \\
\hline 7 & TORTILLA & POLLO & CAFÉ \\
\hline
\end{tabular}




\begin{tabular}{|l|l|l|l|}
\hline 8 & VERDURA & PESCADO & PESCADO \\
\hline 9 & CARNE & CAFÉ & PATATA \\
\hline 10 & PAN & COCA-COLA & MANZANA \\
\hline
\end{tabular}

Cuadro 7: Alimentos y bebidas: comparación Eslovenia/Salamanca/Finlandia

La riqueza léxica de «La ciudad» y el tema disperso han resultado en 6 unidades comunes del conjunto, 8 entre Eslovenia y Salamanca, 7 entre Eslovenia y Finlandia. Llama la atención la palabra más disponible de los tres grupos: calle. Podemos especular que la visión global del mundo y del concepto de la ciudad ha contribuido a la competencia léxica tan homogénea. Esto nos lleva a la conclusión de que el concepto de ciudad no depende mucho del origen geográfico o cultural del informante.

\begin{tabular}{|l|l|l|l|}
\hline RANGO & ESLOVENIA (14.34) & SALAMANCA (17.84) & FINLANDIA (8.00) \\
\hline 1 & CALLE & CALLE & CALLE \\
\hline 2 & COCHE & COCHE & CASA \\
\hline 3 & CASA & TIENDA & COCHE \\
\hline 4 & GENTE & EDIFICIO & TIENDA \\
\hline 5 & TIENDA & PLAZA & ESCUELA \\
\hline 6 & PARQUE & IGLESIA & PARQUE \\
\hline 7 & ESCUELA & PARQUE & IGLESIA \\
\hline 8 & AUTOBÚS & CASA & GENTE \\
\hline 9 & PLAZA & GENTE & SUPERMERCADO \\
\hline 10 & TRÁFICO & AUTOBÚS & MERCADO \\
\hline
\end{tabular}

Cuadro 8: La ciudad: comparación Eslovenia/Salamanca/Finlandia

Al contrario de «La ciudad», en el centro «El campo» sólo tres de las 10 palabras más disponibles aparecen en las tres listas: árbol, animal y vaca. Esta gran diferencia entre el centro de interés «La ciudad» $\mathrm{y}$ «El campo» parece muy interesante. A pesar de que los dos centros resultan bastante abiertos, en «La ciudad» el índice de cohesión entre las primeras respuestas es muy alto y muy bajo en el centro «El campo». Una posible explicación la podemos encontrar en la geografía. Es verdad que todas las grandes ciudades en Europa (como también en otras partes del mundo) se parecen mucho, así que resulta lógico que todos pensemos primero en las calles, coches, tiendas, plazas, autobuses etc. Mientras que la geografía del campo es tan diversa, que la imagen del campo puede variar bastante de una persona a otra. Por esto es posible que en Eslovenia, donde prevalece la ganadería, pensemos primero en los animales, sobre todo en las vacas o los caballos. Sin embargo, en cuanto a la búsqueda de las razones para la similar o diversa competencia léxica básica entre varias zonas de cotejo, se trata sólo de suposiciones porque, como ya 
lo indica Samper Hernández (2002) en su trabajo, necesitaríamos una nueva investigación que lo explicara.

Como en otros centros, también en éste en la lista eslovena hay asociaciones en forma de adjetivos (aproximadamente un 15\%) y verbos (13\%). El adjetivo más disponible verde se encuentra en la posición 7 y refleja la característica típica del campo esloveno. En Salamanca verde aparece en la posición 49. Los verbos más disponibles son trabajar (la posición 26) y caminar (46), en Finlandia cultivar (14). En Salamanca el mismo verbo (el primer verbo en la lista salmantina) no aparece hasta la posición 117.

\begin{tabular}{|l|l|l|l|}
\hline RANGO & ESLOVENIA (11.22) & SALAMANCA (11.11) & FINLANDIA (5.6) \\
\hline 1 & VACA & ÁRBOL & ÁRBOL \\
\hline 2 & ANIMAL & ANIMAL & BOSQUE \\
\hline 3 & NATURALEZA & RÍO & ANIMAL \\
\hline 4 & CAMPESINO & HIERBA & CASA DE CAMPO \\
\hline 5 & CABALLO & FLOR & LAGO \\
\hline 6 & ÁRBOL & MONTAÑA & TRACTOR \\
\hline 7 & VERDE & LAGO & VACA \\
\hline 8 & BOSQUE & VACA & CABALLO \\
\hline 9 & LECHE & TIERRA & CAMPO \\
\hline 10 & PERRO & CASA & FLOR \\
\hline
\end{tabular}

Cuadro 9: El campo: comparación Eslovenia/Salamanca/Finlandia

Debido al carácter limitado de este centro, 9 de los 10 medios de transporte coinciden en los tres grupos, por lo tanto este centro es el que más similitudes tiene. En la lista eslovena la palabra más frecuente es bicicleta que aparece en el $90 \%$ de los casos, pero la palabra más disponible es coche. Aunque la mayoría de las palabras más disponibles en este centro de interés son los tipos básicos de transporte actual que representan los medios de tierra, aire y agua, encontramos en la lista eslovena también caballo, que refleja la realidad eslovena más que el metro, ya que este medio de transporte en Eslovenia no existe. Esto no quiere decir que los alumnos eslovenos no conozcan el concepto y la palabra metro, pero como no es parte de su vida cotidiana, no la evocan de su memoria. Caballo aparece en la posición 13 en Salamanca y en la 17 en Finlandia. Entre las asociaciones de los alumnos eslovenos se encuentran también varios verbos como viajar, conducir o manejar, palabras en relación con los accidentes como muerte, doctor y hospital, otras palabras en relación con el tráfico como semáforo o embotellamiento. Como excepción, entre las asociaciones encontramos también dos adverbios: lejos y cerca. También la lista salmantina contiene algunos verbos: andar, correr, patinar, subir, bajar. En Finlandia no se encuentra ningún verbo entre las primeras 38 palabras más disponibles, a las que hemos tenido acceso. 


\begin{tabular}{|l|l|l|l|}
\hline RANGO & ESLOVENIA $(10.78)$ & SALAMANCA (11.6) & FINLANDIA (5.9) \\
\hline 1 & COCHE & COCHE & COCHE \\
\hline 2 & BICICLETA & AUTOBÚS & AUTOBÚS \\
\hline 3 & AUTOBÚS & TREN & TREN \\
\hline 4 & AVIÓN & AVIÓN & AVIÓN \\
\hline 5 & TREN & BICICLETA & BICICLETA \\
\hline 6 & PIE & BARCO & BARCO \\
\hline 7 & BARCO & TAXI & TAXI \\
\hline 8 & MOTO & METRO & METRO \\
\hline 9 & TAXI & PIE & A PIE \\
\hline 10 & CABALLO & MOTO & MOTO \\
\hline
\end{tabular}

Cuadro 10: Medios de transporte: comparación Eslovenia/Salamanca/Finlandia

Comparando los resultados del centro «Los animales», vemos que seis de las diez palabras más disponibles se repiten en las tres investigaciones. Sin embargo, más que el número resulta interesante la posición tan parecida de las palabras que se repiten. Podemos observar también que las palabras que indican animales domésticos son más disponibles que las que indican animales salvajes. Encontramos también verbos como cuidar, pasear o jugar, adjetivos como pequeño, peligroso o fiel, colores, productos derivados de los animales como huevo o leche, palabras que se relacionan con la caza como violencia, pistola o cazador. En otras dos listas no hay tanta variedad de asociaciones expresadas por adjetivos o verbos.

\begin{tabular}{|l|l|l|l|}
\hline RANGO & ESLOVENIA (11.63) & SALAMANCA (13.95) & FINLANDIA (7.4) \\
\hline 1 & PERRO & PERRO & PERRO \\
\hline 2 & GATO & GATO & GATO \\
\hline 3 & VACA & VACA & CABALLO \\
\hline 4 & CABALLO & PÁJARO & VACA \\
\hline 5 & PÁJARO & CABALLO & TORO \\
\hline 6 & TORO & TORO & PEZ \\
\hline 7 & ARAÑA & SERPIENTE & PÁJARO \\
\hline 8 & OVEJA & ELEFANTE & SERPIENTE \\
\hline 9 & PEZ & CERDO & ZORRO \\
\hline 10 & POLLO & LEÓN & MONO \\
\hline
\end{tabular}

Cuadro 11: Los animales: comparación Eslovenia/Salamanca/Finlandia 
En cuanto al léxico relacionado con «Juegos y distracciones», los tres grupos tienen 3 palabras en común. Entre Eslovenia y Salamanca hay 4, entre Eslovenia y Finlandia hay 7, y entre Salamanca y Finlandia hay 5 palabras comunes. El centro es bastante abierto y ofrece una gran variedad de vocablos. Considerando esto, no sorprenden las similitudes un poco inferiores. Lo que sí sorprende en este centro es la aparición de los verbos ya entre los primeros diez vocablos más disponibles: jugar, nadar, leer, bailar, correr, esquiar. Entre los 297 vocablos que escribieron los alumnos eslovenos, un 25\% de ellos son verbos. Hay muchas discrepancias en algunos vocablos entre informantes de los tres grupos; por ejemplo golf tiene la posición 11 en Salamanca, 17 en Finlandia, pero sólo 77 en Eslovenia. Un caso muy parecido es hockey con la posición 11 en Finlandia, 15 en Salamanca, y 173 en Eslovenia. Lo contrario ocurre con ordenador, que es mucho más disponible en Eslovenia que entre los estudiantes extranjeros de Salamanca y Finlandia, tal vez debido esto al estudio esloveno un poco posterior a los otros dos, cuando el ordenador ya se ha convertido en uno de los objetos cotidianos principales de los jóvenes.

\begin{tabular}{|l|l|l|l|}
\hline RANGO & ESLOVENIA (14.01) & SALAMANCA (11.11) & FINLANDIA (6.3) \\
\hline 1 & FÚTBOL & FÚTBOL & FÚTBOL \\
\hline 2 & BALONCESTO & TENIS & TENIS \\
\hline 3 & MÚSICA & BALONCESTO & TELEVISIÓN \\
\hline 4 & JUGAR & (JUGAR A LAS) CARTAS & CINE \\
\hline 5 & NADAR & LEER & BAILAR \\
\hline 6 & TELEVISIÓN & BAILAR & TEATRO \\
\hline 7 & CINE & NADAR & JUGAR \\
\hline 8 & TENIS & BEISBOL & MÚSICA \\
\hline 9 & DEPORTE & CORRER & LEER \\
\hline 10 & VOLEIBOL & ESQUIAR & NADAR \\
\hline
\end{tabular}

Cuadro 11: Juegos y distracciones: comparación Eslovenia/Salamanca/Finlandia

En «Profesiones y oficios» es elevado el nivel de coincidencia en las primeras dos posiciones. Mientras hay 4 palabras en común para los tres grupos, la coincidencia es un poco más alta entre Eslovenia y Salamanca y Eslovenia y Finlandia -5 palabras. Las primeras dos profesiones no sólo coinciden en las posiciones, sino también en la gran ventaja que tiene profesor en cuanto al índice de disponibilidad alto (más de 0.8 en Salamanca y Finlandia, 0.65 en Eslovenia), le sigue médico con un salto cuantitativo -el índice es de 0.4 en Finlandia y un poco más de 0.3 en Salamanca y Eslovenia. Claro está que si la recogida de los datos para la disponibilidad léxica se lleva a cabo en un contexto escolar, el profesor será la primera asociación. 


\begin{tabular}{|l|l|l|l|}
\hline RANGO & ESLOVENIA (12.94) & SALAMANCA (12.66) & FINLANDIA (7.5) \\
\hline 1 & PROFESOR & PROFESOR & PROFESOR \\
\hline 2 & MÉDICO & MÉDICO & MÉDICO \\
\hline 3 & ACTOR & ABOGADO & POLICÍA \\
\hline 4 & DOCTOR & ESTUDIANTE & VENDEDOR \\
\hline 5 & DIRECTOR & CAMARERO & JARDINERO \\
\hline 6 & CANTANTE & POLICÍA & CURA \\
\hline 7 & ACTRIZ & MAESTRO & CAMARERO \\
\hline 8 & CAMARERO & AMA DE CASA & BOMBERO \\
\hline 9 & ABOGADO & SECRETARIO & BAILARÍN \\
\hline 10 & POLICÍA & ENFERMERO & ACTOR \\
\hline
\end{tabular}

Cuadro 12: Profesiones y oficios: comparación Eslovenia/Salamanca/Finlandia

\section{Conclusiones}

Con el léxico disponible en ELE hemos obtenido sobre todo una gran cantidad de sustantivos y sólo algunos verbos y adjetivos -el componente más inestable de la competencia léxica según López Chávez (1994) y otros lingüistas- que además del léxico básico al que le pertenecen las palabras gramaticales y la inmensa mayoría de los verbos y adjetivos, forman el léxico fundamental de los usuarios de ELE. El predominio de los sustantivos en la disponibilidad léxica se debe probablemente también a una característica del lexicón mental -la palabra estímulo suele provocar la asociación de la misma clase gramatical. Tampoco puede negarse que los sustantivos son más imaginables (Singleton, 1999: 142). Suponemos que la situación de que en las listas eslovenas podemos encontrar más verbos y adjetivos que en las listas de Salamanca o Finlandia, puede estar relacionada con las instrucciones que recibieron los informantes.

Las diez palabras más disponibles para cada estímulo o campo semántico demuestran que en la disponibilidad léxica prevalecen las asociaciones paradigmáticas ante las sintagmáticas (i.e. en las primeras posiciones podemos encontrar perro o gato, cuidar o dar de comer tendrían el índice mucho más bajo). Defiende Singleton (1999: 135) que esto es una característica típica de los usuarios adultos de la lengua materna, lo que se extiende también al uso de una lengua extranjera.

En cuanto a la comparación cuantitativa, la producción general de palabras y vocablos en Eslovenia es superior a la de Salamanca y Finlandia. Por otro lado, la coincidencia de los centros más productivos es sorprendente: los informantes de los tres estudios han mostrado mayor productividad de palabras en el campo sobre la comida y bebida, seguido por el campo semántico sobre la ciudad.

En cuanto al léxico convergente o común entre los tres grupos, partiendo de los primeros diez vocablos más disponibles de los diez centros de interés cotejados en este 
estudio, destacan el centro «Medios de transporte» (9 vocablos comunes), «La ropa» (7 vocablos comunes), «Las partes del cuerpo», «La ciudad»y «Los animales» (6 vocablos comunes). Los centros con menos similitud son los que tienen tres vocablos comunes: «La casa», «El campo» y «Juegos y distracciones». Estos resultados no confirman totalmente lo que afirma Carcedo González (2000b: 182): «la similitud entre los dos grupos de informantes es mayor cuando el número de clases lógicas que componen el estímulo (centro de interés) es más limitado (partes del cuerpo, la ropa, partes de la casa, medios de transporte) y menor cuando registran más amplitud semántica (el campo, la ciudad, juegos y distracciones)», siendo la ciudad una gran excepción con mucha similitud entre los tres grupos observados, a pesar de ser un campo semántico abierto. Suponemos que esto se debe al concepto universal de ciudad. La heterogeneidad léxica de los centros la casa, el campo y juegos y distracciones confirma lo que Mackey expresó ya hace varias décadas: «La disponibilidad es una medida de las diferencias culturales» (Mackey en Carcedo Gónzalez, 2000: 54). Los vocablos de muchos campos muestran una estrecha relación con las peculiaridades culturales.

Este cotejo pone de relieve la similar competencia léxica básica que tienen los estudiantes de ELE, a pesar de tener lenguas maternas tan diversas y vivir en culturas muy diferentes.

En los tres grupos de los informantes de ELE prevalece la selección léxica más cercana a la lengua estándar y peninsular, puesto que la mayoría de los informantes son europeos.

Las investigaciones de la disponibilidad léxica en ELE simplemente han adaptado -e incluso adoptado- la metodología diseñada para la disponibilidad léxica en el español como lengua materna. Se han descartado algunas variables (el nivel sociocultural, la instrucción pública o privada, la procedencia urbana o rural) y en algunos casos añadido otras (el conocimiento de otras lenguas o el nivel de conocimiento), pero lo que verdaderamente hace falta es tomar en cuenta las características del input de ELE, de las cuales depende la competencia léxica: el tipo de metodología, el input por parte del profesor y de los manuales sobre todo en los estudiantes que no estudian ELE en inmersión. Una vez establecidos los criterios para cotejar la disponibilidad léxica en ELE, se podrán sacar conclusiones más exhaustivas y finales. Por eso, en este artículo no hemos tratado tanto de proporcionar conclusiones definitivas al problema planeado como de dar unas muestras sobre una línea de investigación que consideramos muy fructífera, sobre todo para la enseñanza de léxico de ELE.

\section{BIBLIOGRAFÍA}

Alba, O. (1995): Léxico disponible de la República Dominicana. Santo Domingo: Pontificia Universidad Católica «Madre y Maestra».

Bartol, J. A., Hernández, N. (2003): Dispolex: http://www.dispolex.com 
Benítez Pérez, P. (1992): «Disponibilidad léxica en la zona metropolitana de Madrid». En: Boletín de la Academia Puertorriqueña de la Lengua Española, 1, 1, 71-102.

Benítez Pérez, P. (1994): «¿Qué vocabulario hay que enseñar en las clases de español como lengua extranjera?». En: Miquel, L. y Sans, N. (eds.): Didáctica del Español como Lengua Extranjera. Madrid: Cuadernos del Tiempo Libre, 9-12.

Carcedo González, A. (2000): Disponibilidad léxica en español como lengua extranjera: el caso finlandés (estudio del nivel preuniversitario y cotejo con tres fases de adquisición). Turku: Universidad de Turku.

Carcedo González, A. (2001): Léxico disponible de Asturias. Turku: Universidad de Turku.

Frey Pereyra, Mª L. H. (2007): «Disponibilidad léxica y escritura del español como lengua extranjera: propuesta de comparación de dos corpus» En: Interlingüística, n¹7, 366-373.

Galloso Camacho, M. V. (1998): El léxico disponible en el nivel preuniversitario (provincia de Zamora) (memoria de licenciatura). Salamanca: Universidad de Salamanca.

Galloso Camacho, M. V. y Prado Aragonés, J. (2005): «La estructura estadística del léxico disponible de informantes nativos e informantes de ELE». En: Castillo Carballo, Ma A. (ed.), Las gramáticas y los diccionarios en la enseñanza del español como segunda lengua: Deseo y realidad. Actas de XV Congreso Internacional de ASELE. Sevilla: Universidad de Sevilla, 370-375.

García Marcos, F. y Mateo, Ma V. (2000): La selección de materiales léxicos en la enseñanza de lenguas extranjeras. Evaluación y propuestas desde la disponibilidad léxica, MS.

González Martínez, A. (2002): La disponibilidad léxica de los alumnos preuniversitarios de la provincia de Cádiz. Cádiz: Universidad de Cádiz.

Izquierdo Gil, M ${ }^{\mathrm{a}} \mathrm{C}$. (2005): La selección del léxico en la enseñanza del español como lengua extranjera. Málaga: ASELE, Colección Monografías nº 8.

Kranjc, M. (2009): Disponibilidad léxica en alumnos eslovenos en español como lengua extranjera. (memoria de licenciatura). Ljubljana: Universidad de Ljubljana.

López Chávez, J. (1993): El léxico disponible de escolares mexicanos. México: Editorial Alhambra Mexicana.

López Chávez, J. (1994): «Comportamiento sintáctico de algunos verbos ordenados según su grado de disponibilidad léxica». En: Revista de estudios de adquisición de la lengua española. número 1. Alcalá de Henares: Universidad de Alcalá, 67-84.

López Morales, H. (1973): Disponibilidad léxica de los escolares de San Juan. MS inédito.

López Morales, H. (1999): Léxico disponible de Puerto Rico. Madrid: Arco Libros.

Mateo García, M. V. (1998): Disponibilidad léxica en el c.o.u. almeriense. Estudio de estratificación social. Almería: Universidad de Almería.

Nation, I. S. P. (1990): Teaching and Learning vocabulary. Boston: Heinle \& Heinle Publishers.

Nation, I. S. P. (2001): Learning vocabulary in another language. Cambridge: Cambridge University Press. 
Prado Aragonés, J. y Galloso Camacho, Ma V. (2005): Léxico disponible de Huelva. Nivel preuniversitario. Huelva: Universidad de Huelva.

Rodríguez Muñoz; F. J. y Muñoz Hernández, I. O. (2009): «De la disponibilidad a la didáctica léxica». En: Tejuelo, nº4. Almería: Universidad de Almería, 8-18.

Samper, J. A. y Hernández, C. E. (1997): «El estudio de la disponibilidad léxica en Gran Canaria: datos iniciales y variación sociolingüística». En: Contribuciones al estudio de la lingüística hispánica (Homenaje al Profesor Ramón Trujillo), Vol. II. Santa Cruz de Tenerife: Montesinos, 229-239.

Samper Hernández, M. (2002): Disponibilidad léxica en alumnos de español como lengua extranjera. Málaga: ASELE, Colección Monografías nº 4.

Sánchez Gómez, C. (2003): Disponibilidad léxica en alumnos de español como lengua extranjera. Tesina inédita. Huelva: Universidad de Huelva.

Singleton, D. (1999): Exploring the Second Language Mental Lexicon. Cambridge: Cambridge University Press.

Valencia, A. y Echeverría, M. (1999): Disponibilidad léxica en estudiantes chilenos. Santiago de Chile: Universidad de Chile-Universidad de Concepción.

\section{LEKSIKALNA RAZPOLOŽLJIVOST V ŠPANŠČINI KOT TUJEM JEZIKU: PRIMERJAVA RAZISKAV IZVEDENIH V SLOVENIJI, SALAMANCI IN NA FINSKEM}

Ključne besede: leksikalna zmožnost, leksikalna razpoložljivost, temeljno besedišče, španščina kot tuji jezik

Avtorica najprej predstavi pojem leksikalne razpoložljivosti, ki pomeni besedišče, ki si ga govorec najprej prikliče v povezavi z določeno temo. Nato predstavi raziskavo o leksikalni razpoložljivosti, ki jo je izvedla med slovenskimi dijaki ob koncu njihovega tretjega leta učenja španščine kot tujega jezika. Rezultate raziskave, ki so vezani na deset semantičnih polj, primerja z raziskavama izvedenima med tečajniki španščine kot tujega jezika v Salamanci in finskimi dijaki v Turkuju. Z uporabo leksikalno-statističnega programa Dispolex avtorica v obliki tabel prikaže, da si tuji govorci španščine različnih maternih jezikov prikličejo na določeno temo zelo podobne besede. Ugotavlja, v katerih semantičnih poljih so anketiranci najbolj oz. najmanj produktivni in kje je leksikalno ujemanje med njimi večje oz. manjše. Za bolj poglobljene zaključke o leksikalni razpoložljivosti v španščini kot tujem jeziku se je izkazalo, da bi potrebovali več podatkov o načinu poučevanja in o učbenikih, ki so jih anketirani pred tem uporabljali. 